\title{
Epidermal stem cell dynamics
}

Maya Sieber-Blum*

\begin{abstract}
Wong and Reiter have explored the possibility that hair follicle stem cells can give rise to basal cell carcinoma (BCC). They expressed in mice an inducible human BCC-derived oncogenic allele of Smoothened, SmoM2, under the control of either the cytokeratin 14 (K14) or cytokeratin 15 (K15) promoter. Smoothened encodes a G-protein-coupled receptor protein in the hedgehog pathway, the misregulation of which is implicated in BCC and other human cancers. Chronic injury is thought to be a contributing factor. The authors used K14 as a marker for stem cells in the basal layer of the epidermis and $\mathrm{K} 15$ as a marker for epidermal stem cells in the bulge of hair follicles. Upon activation, K14 construct-bearing mice readily formed BCC-like tumours, whereas this was not the case in K15:SmoM2carrying mice. Upon wounding the epidermis, however, there was widespread BCC-like tumour formation in the skin of K15:SmoM2 mice. The authors conclude that wounding recruited bulge epidermal stem cells to the surface, allowing the cells to escape quiescence in the stem cell niche and to arrive in an environment where the hedgehog pathway becomes activated and therefore tumorigenesis is elicited. While this is a provocative result and the authors' conclusion may well be correct, there are alternative explanations.
\end{abstract}

This commentary relates to the paper by Wong and Reiter [1], who report that basal cell carcinoma (BCC) can originate from the follicular bulge. Cytokeratins heteropolymerise, with an acidic (type I) cytokeratin binding a neutral-basic (type II) cytokeratin. Cytokeratin 14 (K14) forms heterodimers with cytokeratin 5 . In contrast, less is known about cytokeratin 15 (K15) and there is no known K15 binding partner (reviewed by [2]). During embryogenesis, postnatally, during wounding and in

\footnotetext{
*Correspondence: maya.sieber-blum@ncl.ac.uk

Institute of Genetic Medicine and Northeast England Stem Cell Institute, Newcastle University, Centre for Life, Central Parkway, Newcastle upon Tyne, NE1 3BZ, UK
}

tumourigenesis, cytokeratins are differentially expressed in the basal layer of the mouse epidermis and in the hair follicle [2]. There is a relative lack of consensus among investigators, however, of marker specificity in terms of unequivocally identifying different subsets of stem cell.

It has been well established that K14 is expressed in the basal layer of the epidermis in postnatal mouse skin and can therefore be considered a marker for stem cells/ progenitors in the basal layer of the epidermis [3]. K14 is, however, significantly less useful for distinguishing between progenitor cells in the basal layer and follicular bulge stem cells, as K14-expressing cells have also been described in the bulge of hair follicles (see, for example, $[4,5])$.

Conversely, while K15 has been used as a marker for follicular stem cells in the bulge, K15 has been reported to also be expressed in the basal layer of the epidermis and to thus mark the least differentiated cells in the neonatal epidermis and adult bulge [6]. Likewise, in a careful recent study in which peptide-specific antibodies to K15 were used, it has been found that K15immunoreactive cells are not exclusively limited to the follicular bulge, but that $\mathrm{K} 15$ is also expressed in cells of the basal layer of the mouse epidermis during embryogenesis and throughout adulthood [2]. Thus, during the time of SmoM2 activation in the study by Wong and Reiter [1], at 7.5 weeks of age, K15 is expressed not only in cells of the bulge but also by at least a subset of progenitor cells in the basal layer of the epidermis. Furthermore, K15 expression is down-regulated during wounding and in tumourigenesis. The lack of nichespecific gene expression and the dynamic changes in gene expression patterns during development, during the hair cycle, and in diseased skin poses a problem that consistently restricts interpretation of data in this field of investigation.

It is well established that bulge stem cells respond rapidly upon wounding and are recruited to the damaged area to seal the injury temporarily and in order to be replaced later on by basal layer-derived cells [7].

Wong and Reiter [1] have addressed the issue of K14 and $\mathrm{K} 15$ expression patterns in the two locations by quantifying $\beta$-galactosidase-expressing bulge follicular cells and cells in the epidermal basal layer in K14:SmoM2:LacZ and K15:SmoM2:LacZ mice. They have 
arrived at the same conclusion, that $\mathrm{K} 14$ and $\mathrm{K} 15$ are expressed by cells in both locations, albeit, as expected, in reciprocal ratios. The authors nevertheless suggest that wounding in the K15:SmoM2 mouse recruits the tumourprone bulge stem cells to the wounded area where the hedgehog pathway will be disinhibited, thus allowing tumourigenesis to be induced and to progress. While this may well be the case, strictly speaking the experiments shown in this study do not allow this conclusion. While known to occur in normal mice, migration of constructexpressing cells per se has not been shown in the current study. The data are limited to evidence of $\beta$-galactosidaseexpressing cells in the basal layer of the epidermis. The cells could have immigrated from the bulge, but it is equally possible that they have been resident in the basal layer even prior to wounding and construct activation. Moreover, while admittedly difficult and possibly not achievable, a rigorous experimental design would not only show the dynamics of cell migration in these animal models of BCC, but also include as a control experiment the inhibition of cell migration from the bulge to the wound. In the latter case no tumour formation would be expected if the authors' hypothesis were correct.

BCCs come in many variations - nodular basal-cell carcinoma (classic basal-cell carcinoma), cystic basal-cell carcinoma, cicatricial basal-cell carcinoma, infiltrative basal-cell carcinoma, micronodular basal-cell carcinoma, superficial basal-cell carcinoma, and pigmented basalcell carcinoma. Due to this diversity of BCC, the most likely scenario is that $\mathrm{BCC}$ can have multiple origins that, depending on the specific type of tumour, originate from progenitor cells in the basal layer or the bulge. The existence of pigmented BBCs is intriguing, as neural crest-derived stem cells reside in the bulge of hair follicles [8] and neural crest-derived melanocyte precursors in the matrix differentiate into pigment cells that inject their melanosomes into keratinocytes and thus give hair its colour.
Abbreviations

BBC, basal cell carcinoma; K, cytokeratin; Smo, smoothened.

Competing interests

The author declares no competing interests.

\section{Author information}

MSB's research expertise is in the mechanisms that regulate differentiation of embryonic neural crest stem cells and in the area of somatic neural crestderived stem cells in hair follicles. Her current position is Professor of Stem Cell Sciences in the Institute of Genetic Medicine at Newcastle University. She is a member of the UKNSCN, ISSCR and SSCN and the Society for Neuroscience.

\section{Acknowledgements}

Supported by Medical Research Council Grant 22358.

Published: 29 June 2011

\section{References}

1. Wong SY, Reiter JF: Wounding mobilizes hair follicle stem cells to form tumors. Proc Natl Acad Sci U S A 2011, 108:4093-4098.

2. Troy T-C, Arabzadeh A, Turksen K: Re-assessing K15 as an epidermal stem cell marker. Stem Cell Rev Rep 2011. DOI 10.1007/s12015-011-9243-9.

3. Vasioukhin V, Degenstein L, Wise B, Fuchs E: The magical touch: Genome targeting in epidermal stem cells induced by tamoxifen application to mouse skin. Proc Natl Acad Sci U S A 1999, 96:8551-8556.

4. Tumbar T, Guasch G, Greco V, Blanpain C, Lowry WE, Rendl M, Fuchs E: Defining the epithelial stem cell niche in skin. Science 2004, 303:359-363.

5. Rendl M, Lewis L, Fuchs E: Molecular dissection of mesenchymal-epithelial interactions in the hair follicle. PLOS Bio/ 2005, 3:e331.

6. Liu Y, Lyle S, Yang Z, Cotsarelis G: Keratin 15 promoter targets putative epithelial stem cells in the hair follicle bulge. J Invest Dermato/ 2003, 121:963-968.

7. Ito M, Liu Y, Yang Z, Nguyen J, Liang F, Morris RJ, Cotsarelis G: Stem cells in the hair follicle bulge contribute to wound repair but not to homeostasis of the epidermis. Nat Med 2005, 11:1351-1354.

8. Clewes O, Narytnyk A, Gillinder KR, Loughney AD, Murdoch AP, Sieber-Blum M: Human epidermal neural crest stem cells (hEPI-NCSC) - characterization and directed differentiation into osteocytes and melanocytes. Stem Cell Rev Rep 2011. DOI 10.1007/s12015-011-9255-5.

doi:10.1186/scrt70

Cite this article as: Sieber-Blum M: Epidermal stem cell dynamics. Stem Cell Research \& Therapy 2011, 2:29. 